\title{
PAINÉIS DE MADEIRA AGLOMERADA DE RESÍDUOS DA LAMINAÇÃO DE DIFERENTES PROCEDÊNCIAS DE Eucalyptus grandis, Eucalyptus saligna E Eucalyptus cloeziana
}

\author{
José Benedito Guimarães Júnior ${ }^{1}$, Lourival Marin Mendes², Rafael Farinassi Mendes ${ }^{3}$, Fábio Akira Mori
}

(recebido: 5 de agosto de 2009; aceito: 30 de junho de 2011)

RESUMO: Com este trabalho, objetivou-se fazer a avaliação de 15 procedências de espécies de Eucalyptus cloeziana, Eucalyptus grandis e Eucalyptus saligna, com idade de 31 anos, sendo que esse teste foi instalado no campus da Universidade Federal de Lavras. Foram fabricados 6 painéis aglomerados para cada procedência, a partir dos resíduos gerados pela laminação desse material. Foi utilizado o adesivo uréia-formaldeído com $8 \%$, e parafina a 1\%, ambos com base nos seus respectivos teores de sólidos. Os painéis foram prensados à temperatura de $160^{\circ} \mathrm{C}$, pressão específica de $3,92 \mathrm{MPa}$ por tempo de 8 minutos. A partir dos resultados concluiuse que: em absorção de água, destacou-se a espécie Eucalyptus saligna; em inchamento em espessura, a procedência 43 mostrou melhor desempenho dentro da espécie de Eucalyptus grandis; foram obtidos resultados superiores para Eucalyptus cloeziana no que se refere à compressão e MOE; para Eucalyptus grandis, a procedência de número 10695 apresentou melhores propriedades de MOE e MOR. De forma geral, a procedência 10.695 e as espécies de Eucalyptus grandis foram as que apresentaram maiores potenciais para a produção de painéis aglomerados.

Palavras-chave: Painéis aglomerados, eucalipto, procedências.

\section{WOOD PATICLEBOARDS MADE FROM RESIDUES OBTAINED IN THE VENEER PRODUCTION OF EUCALYPT SPECIES AND PROVENANCES}

\begin{abstract}
This work aimed the evaluation of 15 (fifteen) provenances of three eucalypt species: Eucalyptus cloeziana, Eucalyptus grandis and Eucalyptus saligna. The trees were 31 years old and the test was developed in the campus of the Universidade Federal de Lavras. Six particleboards for each provenance were made by using the residues generated in the veneer production of this material. It was used urea-formaldehyde at $8 \%$ resin level and paraffin at 1\%, both according to their respective solid content. The boards were produced by using a pressing cycle with temperature of $160{ }^{\circ} \mathrm{C}$, specific pressure of $40 \mathrm{kgf} / \mathrm{cm}^{2}$ and pressing time of 8 minutes. From the results, it was concluded that the specie Eucalyptus saligna was the best one in terms of water absorption. The 43 provenance of Eucalyptus grandis showed the best development of thickness swelling. Superior results of compression and elastic modulus were found to Eucalyptus cloeziana. The provenance of number 10695 of the specie Eucalyptus grandis presented the best results for elastic and rupture modulus. In general, the provenance 10695 and species of Eucalyptus grandis showed the greatest potential for production of particleboard.
\end{abstract}

Key words: Particleboards, eucalypt, provenances.

\section{INTRODUÇÃO}

Os painéis aglomerados caracterizam-se pela transformação da madeira em pequenas partículas que, secas e misturadas com adesivos sintéticos termofixos e distribuídas aleatoriamente entre si, são prensadas sob calor e pressão, gerando um painel particulado (MOSLEMI, 1974; TSOUMIS, 1991). De acordo com esse conceito, pode-se dizer que todo e qualquer material lignocelulósico tem potencial de ser utilizado como matéria-prima para a fabricação de chapas de partículas.
Nesse sentido confere a oportunidade de utilização de resíduos florestais e agroindustriais na sua produção.

De acordo com Kubler (1980) o painel aglomerado nasceu da necessidade de se utilizar resíduos lignocelulósico, uma vez que, próximo a Segunda Guerra Mundial, houve uma escassez de madeira sólida. Desde então esse painel vem sendo produzido e utilizado em grande escala. Dados da Food and Agriculture Organization - FAO (2011) apontam que a produção mundial cresceu de 79.366.400 para 93.949.802 metros cúbicos, entre os anos de 1999 e 2009.

\footnotetext{
'Engenheiro Florestal, Professor Dr. em Ciência e Tecnologia da Madeira - Departamento de Engenharias - Universidade Federal do Piauí/UFPI BR135, Km 03 - Bom Jesus, PI - 64.900-000 - jbguimaraesjr@hotmail.com

${ }^{2}$ Engenheiro Florestal, Professor Dr. em Engenharia Florestal - Departamento de Ciências Florestais - Universidade Federal de Lavras - Cx. P. 3037 37200-000 - Lavras, MG - lourival@dcf.ufla.br

${ }^{3}$ Engenheiro Florestal, Doutorando em Ciência e Tecnologia da Madeira - Departamento de Ciências Florestais/DCF - Universidade Federal de Lavras/UFLA - Cx. P. 3037 - 37200-000 - Lavras, MG - rafaelfarinassi@gmail.com

${ }^{4}$ Engenheiro Florestal, Professor Dr. em Ciências Florestais - Departamento de Ciências Florestais - Universidade Federal de Lavras - Cx. P. 3037 37200-000 - Lavras, MG - morif@dcf.ufla.br
}

Cerne, Lavras, v. 17, n. 4, p. 443-452, out./dez. 2011 
As indústrias de base florestal no Brasil geram um volume significativo de resíduos durante as fases operacionais, desde a exploração florestal até o produto final. De acordo com Brito et al. (2006), as indústrias de madeira serrada, lâminas e compensados geraram resíduos da ordem de 19.255.000 metros cúbicos por ano; representando $50,7 \%$ do volume original de toras.

De acordo com Fagundes (2003), a utilização de resíduos gerados pela indústria madeireira, tais como: serragem, maravalha, resíduos da indústria de papel, roloresto e resíduos de laminação, dentre outros contribuem para aumentar à sustentabilidade de utilização das florestas, de forma especial as plantadas, além de gerar benefícios sociais. Bufalino (2010) enfatiza ainda que essa prática é especialmente interessante em locais onde há escassez de madeira.

De acordo com Iwakiri et al. (2000), a grande maioria dos resíduos provenientes de processamento mecânico da madeira é utilizada para geração de energia para fins industriais e domésticos. Todavia, apesar dos benefícios sociais, essa forma de utilização agrega muito pouco valor ao produto final, sendo necessárias outras formas alternativas de utilização, como a produção de painéis de madeira aglomerada.

Com relação aos estudos de espécies e procedências, pode-se salientar que esta vem ganhando importância, tendo em vista a variabilidade natural e a adequação destes aos diversos usos, principalmente no que se refere aos painéis reconstituídos. As variações existentes entre espécies, procedências, famílias e clones podem oferecer uma oportunidade de se alterar características importantes na madeira, na busca de se produzir matéria-prima com qualidade adequada na manufatura de diferentes produtos à base de madeira. As características mais importantes e consideradas limitantes a viabilização do emprego do eucalipto se encontram sob moderado a alto controle genético, tornando possível alterar seus valores, a fim de encontrar a qualidade necessária para o uso industrial.

No que se refere às propriedades da madeira, aquela que é considerada a mais importante relacionada ao material genético, é a densidade, sendo fator determinante na utilização da matéria-prima para a produção de painéis (KELLY, 1977). A indústria, normalmente, utiliza madeiras com densidades inferiores a $0,60 \mathrm{~g} / \mathrm{cm}^{3}$ (MALONEY, 1993), porque as chapas devem ter densidade de 5 a $40 \%$ superior à densidade da madeira empregada na sua fabricação (HRÁZSKÝ; KRAL, 2003, citados por CABRAL et al., 2007).

Cerne, Lavras, v. 17, n. 4, p. 443-452, out./dez. 2011
A relação entre a densidade da chapa e a densidade da madeira, é denominada de razão de compactação, exercendo um efeito marcante nas propriedades dos painéis particulados, tendo em vista o processo de densificação do material, para consolidação do painel até a espessura final (KELLY, 1977; MALONEY, 1993; MOSLEMI, 1974).

Uma densidade mais baixa da madeira, considerando constante a densidade do painel, promove uma razão de compactação mais elevada e, por conseguinte, uma maior superfície de contato entre as partículas em comparação a uma madeira de densidade mais alta (CLOUTIER, 1998). Essa maior quantidade de partículas compactadas a um mesmo volume leva a uma capacidade mais elevada de transmitir esforços. Por consequência as propriedades de flexão estática e tração perpendicular são mais elevadas para chapas confeccionadas com madeira de baixa densidade.

De acordo com Kelly (1977), em painéis com maior razão de compactação, há maior quantidade de partículas de madeira e, consequentemente, maior densificação do painel, resultando em maior inchamento higroscópico da madeira e liberação das tensões de compressão geradas durante o processo de prensagem. Dessa forma, pode-se perceber que as propriedades básicas do material genético são fatores que irão interferir de maneira direta nas propriedades físicas e mecânicas dos painéis de madeira aglomerada.

Nesse sentido, conduziu-se este trabalho, com o objetivo de avaliar a qualidade dos painéis produzidos a partir de resíduos gerados pela laminação de três espécies de eucalipto de diferentes procedências, por meio da mensuração de suas propriedades físicas e mecânicas.

\section{MATERIAL E MÉTODOS}

Neste estudo, foram utilizadas árvores de 10 procedências de Eucalyptus grandis, 3 de Eucalyptus saligna e 2 de Eucalyptus cloeziana, que apresentavam a idade de 31 anos. Foram abatidas 4 árvores por procedência. Estas faziam parte do Programa de Introdução de Espécies e Procedências de Eucalipto, no sul de Minas Gerais (IBDFPRODEPEF). O plantio foi instalado na Escola Superior de Agricultura de Lavras (ESAL), hoje Universidade Federal de Lavras (UFLA). Na seleção foi tomado o cuidado de escolher árvores que apresentassem o fuste mais linear e que possuíssem bom estado fitossanitário.

A cidade de Lavras está localizada à latitude de $21^{\circ}$ 14 'S e longitude $45^{\circ} 00^{\prime} \mathrm{w}$, sua altitude média é de $900 \mathrm{~m}$. 
A precipitação média anual está em torno de $1400 \mathrm{~mm}$ e a temperatura média anual é de $19,4^{\circ} \mathrm{C}$.

Esse material genético foi plantado no espaçamento de $3 \times 2$ metros. Na Tabela 1, encontram-se os valores de diâmetro a altura do peito, medido a 1,30 metros do solo (DAP) e densidade básica para cada procedência.

Foram produzidos 6 painéis para cada procedência avaliada, totalizando 90 painéis aglomerados. As dimensões e a densidade nominal foram, respectivamente, de $480 \mathrm{~mm}$ x $480 \mathrm{~mm}$ x $15 \mathrm{~mm}$ e $0,70 \mathrm{~g} / \mathrm{cm}^{3}$.

$\mathrm{O}$ adesivo utilizado foi a ureia-formaldeído, com teor de sólidos de $65,06 \%$, pH de 8,42, viscosidade de 480 cP e Gel Time de 51 segundos. A aplicação de adesivo foi de $8 \%$ em relação à massa seca das partículas. Da mesma forma, foi aplicada a emulsão de parafina, $1 \%$ em relação à massa seca das partículas.

As partículas "sliver" foram obtidas a partir das perdas geradas pela laminação, no processo de arredondamento da tora, sendo processadas em moinho de martelo. Estas foram peneiradas, sendo utilizadas para manufatura do painel aquelas que ficavam retidas entre as peneira de 10 e 30 mesh. Em seguida, foram secas até o conteúdo de umidade de $3 \%$.
Os painéis foram prensados à temperatura de $160^{\circ} \mathrm{C}$, pressão específica de $3,92 \mathrm{MPa}$ por um período de 8 minutos.

Retiraram-se 4 corpos-de-prova por painel, para avaliação das propriedades de flexão estática - DIN 52362 (DEUTSCHES INSTITUT FUR NORMUNG - DIN, 1982); 4 corpos-de-prova por painel, para compressão - ASTM D1037-34.22 (AMERICAN SOCIETY FOR TESTING AND MATERIAL - ASTM, 2002), 6 corposde-prova por painel, para tração perpendicular - ASTM D1037-28 (ASTM, 1982) e 2 corpos-de-prova por painel, para absorção de água e inchamento em espessura após $2 \mathrm{e}$ 24 horas de imersão - ASTM D1037-100 (ASTM, 1982). Os mesmos foram climatizados à temperatura de $20 \pm 3^{\circ} \mathrm{C}$ e umidade relativa de $65 \pm 5 \%$.

Utilizou-se o delineamento inteiramente casualizado, com 6 repetições, em que os tratamentos foram arranjados em um esquema hierárquico, estudando-se três espécies com diferentes procedências dentro de espécies. Suspeitando-se de que a densidade do painel $(\mathrm{X})$ poderia afetar as suas propriedades, procedeu-se a uma análise de covariância, com as médias ajustadas para a densidade do painel. Utilizouse do teste de médias de Tukey, com 5\% de significância.

Tabela 1 - Dados de DAP e densidade básica do material genético utilizado.

Table 1 -Data of DAP and basic density of the used genetic material.

\begin{tabular}{|c|c|c|c|c|c|c|}
\hline \multirow{3}{*}{ Procedência } & \multicolumn{6}{|c|}{ Espécies } \\
\hline & \multicolumn{2}{|c|}{ E. cloeziana } & \multicolumn{2}{|c|}{ E. grandis } & \multicolumn{2}{|c|}{ E. saligna } \\
\hline & $\begin{array}{l}\text { DAP } \\
(\mathrm{cm})\end{array}$ & $\begin{array}{c}\text { Densidade } \\
\left(\mathrm{g} / \mathrm{cm}^{3}\right)\end{array}$ & $\begin{array}{l}\text { DAP } \\
(\mathrm{cm})\end{array}$ & $\begin{array}{c}\text { Densidade } \\
\left(\mathrm{g} / \mathrm{cm}^{3}\right)\end{array}$ & $\begin{array}{l}\text { DAP } \\
(\mathrm{cm})\end{array}$ & $\begin{array}{c}\text { Densidade } \\
\left(\mathrm{g} / \mathrm{cm}^{3}\right)\end{array}$ \\
\hline 9785 & 34,0 & 0,71 & --- & --- & --- & --- \\
\hline 97852 & 34,0 & 0,75 & --- & --- & --- & --- \\
\hline 43 & --- & --- & 36,0 & 0,56 & --- & --- \\
\hline 45 & --- & --- & 31,0 & 0,59 & --- & --- \\
\hline 47 & --- & --- & 41,8 & 0,60 & --- & --- \\
\hline 48 & --- & --- & 46,5 & 0,58 & --- & --- \\
\hline 8535 & --- & --- & 35,6 & 0,54 & --- & --- \\
\hline 9753 & --- & --- & 50,0 & 0,61 & --- & --- \\
\hline 9789 & --- & --- & 58,0 & 0,62 & --- & --- \\
\hline 10634 & --- & --- & 39,6 & 0,59 & --- & --- \\
\hline 10695 & --- & --- & 38,5 & 0,60 & --- & --- \\
\hline 10696 & --- & --- & 30,0 & 0,61 & --- & --- \\
\hline 7785 & --- & --- & --- & --- & 37,5 & 0,63 \\
\hline 7808 & --- & --- & --- & --- & 42,0 & 0,64 \\
\hline 10698 & --- & --- & --- & --- & 34,2 & 0,57 \\
\hline
\end{tabular}

Cerne, Lavras, v. 17, n. 4, p. 443-452, out./dez. 2011 


\section{RESULTADOS E DISCUSSÃO}

Os valores médios de densidade aparente para os painéis aglomerados estão apresentados na Tabela 2 .

Tabela 2 - Valores médios de densidade do painel, segundo as espécies e a procedência.

Table 2 - Medium values of density of the panel, according to species and provenances.

\begin{tabular}{lccc}
\hline \multirow{2}{*}{ Procedência } & \multicolumn{3}{c}{ Espécies $^{1}$} \\
\cline { 2 - 4 } & $\begin{array}{c}\text { E. cloeziana } \\
\left(\mathrm{g} / \mathrm{cm}^{3}\right)\end{array}$ & $\begin{array}{c}\text { E. grandis } \\
\left(\mathrm{g} / \mathrm{cm}^{3}\right)\end{array}$ & $\begin{array}{c}\text { E. saligna } \\
\left(\mathrm{g} / \mathrm{cm}^{3}\right)\end{array}$ \\
\hline 9785 & $0,61 \mathrm{~A}$ & --- & --- \\
97852 & $0,64 \mathrm{~A}$ & --- & --- \\
43 & --- & $0,57 \mathrm{AB}$ & --- \\
45 & --- & $0,63 \mathrm{~A}$ & --- \\
47 & --- & $0,62 \mathrm{~A}$ & --- \\
48 & --- & $0,60 \mathrm{AB}$ & --- \\
8535 & --- & $0,65 \mathrm{~A}$ & --- \\
9753 & --- & $0,61 \mathrm{AB}$ & --- \\
9789 & --- & $0,63 \mathrm{~A}$ & --- \\
10634 & --- & $0,53 \mathrm{~B}$ & --- \\
10695 & --- & $0,64 \mathrm{~A}$ & --- \\
10696 & --- & $0,62 \mathrm{~A}$ & --- \\
7785 & --- & --- & $0,61 \mathrm{~A}$ \\
7808 & --- & --- & $0,60 \mathrm{~A}$ \\
10698 & --- & --- & $0,64 \mathrm{~A}$ \\
\hline Média Espécies ${ }^{1}$ & $0,62 \mathrm{a}$ & $0,61 \mathrm{a}$ & $0,61 \mathrm{a}$ \\
\hline CV $(\%)$ & & 7,15 & \\
\hline
\end{tabular}

${ }^{1}$ Médias seguidas de mesma letra minúscula, na linha, e maiúscula, na coluna, não diferem entre si, pelo teste Tukey, a $5 \%$ de significância.

Para as procedências de Eucalyptus cloeziana e Eucalyptus saligna, não houve diferenças entre as densidades avaliadas. As procedências de Eucalyptus grandis apresentaram diferenças significativas para a densidade. Entre as espécies, os valores variaram de 0,61 a $0,62 \mathrm{~g} / \mathrm{cm}^{3}$, não diferindo entre si. Os baixos valores de densidade observados, tendo como base aquela nominal $\left(0,70 \mathrm{~g} / \mathrm{cm}^{3}\right)$, podem ser atribuídos à especificidade das condições laboratoriais em relação ao processo industrial, com perdas de materiais durante o manuseio das partículas nas etapas de aplicação de adesivo, formação do colchão e prensagem dos painéis.

Cerne, Lavras, v. 17, n. 4, p. 443-452, out./dez. 2011
As procedências 43 e 10634 de Eucalyptus grandis apresentaram valores de densidade inferiores $0,60 \mathrm{~g} / \mathrm{cm}^{3}$; sendo considerados pela norma CS 236-66 (COMMERCIAL STANDARD - CS, 1968) como painéis de baixa densidade. Os demais foram considerados, por essa mesma norma, como painéis de média densidade. As exigências impostas pela mesma norma para as propriedades físico-mecânicas baseiam-se no valor da densidade dos painéis. Portanto, houve a necessidade de separar essas duas procedências das demais para avaliação.

Os resultados da análise de covariância para absorção de água, inchamento em espessura, tração perpendicular, MOE e MOR, considerando a densidade dos painéis como covariável mostra que os resíduos seguem uma distribuição normal, segundo o teste de Shapiro-Wilk. A regressão linear não significativa indica que não existe relação linear entre essas variáveis e a densidade dos painéis.

Para o teste de compressão, considerando a densidade dos painéis como co-variável mostra que o resíduo, para essa variável analisada, segue uma distribuição normal, segundo o teste de Shapiro-Wilk, p-valor de 0,7425. A regressão linear foi significativa ( $\mathrm{p}$-valor $=0,0088)$. Ou seja, existe uma relação linear entre essa variável e a densidade dos painéis. A estimativa do coeficiente de regressão linear $(\hat{b}=77,68)$ indica que, para o aumento de $0,1 \mathrm{~g} / \mathrm{cm}^{3}$ na densidade dos painéis, há um aumento de $7,768 \mathrm{kgf} / \mathrm{cm}^{2}$ na resistência à compressão.

Os dados da Tabela 3 apresentam os valores observados para as espécies e procedências estudadas com relação à absorção de água após 2 e 24 horas de imersão, respectivamente. Para a absorção de água após 2 horas de imersão, não houve diferença estatística para as procedências de Eucalyptus cloeziana e Eucalyptus saligna. Analisando-se as diferentes procedências de Eucalyptus grandis, nota-se que a 10695 apresentou menor média para absorção de água, com $34,88 \%$, sendo estatisticamente igual a 45, 9753, 9789 e 10634 . A espécie que apresentou menor média para absorção de água foi o Eucalyptus saligna, com 45,66\%, sendo, estatisticamente, igual ao Eucalyptus grandis.

Para a absorção de água após 24 horas, notou-se a mesma tendência observada para 2 horas, as procedências de Eucalyptus cloeziana e Eucalyptus saligna não apresentaram diferenças estatísticas entre suas médias. Para Eucalyptus grandis, a procedência que demonstrou menor valor médio foi a 9789 , com $72,83 \%$. Esta foi estatisticamente igual às de número 43, 45, 47, 48, 9753, 10695 e 10696. Entre as espécies, Eucalyptus saligna apresentou menor valor médio para absorção, após 24 horas de imersão, com 71,16\%, sendo estatisticamente igual à Eucalyptus cloeziana. 
Tabela 3 - Valores médios de absorção de água, em \% em 2 e 24 horas, segundo as espécies e a procedência.

Table 3-Medium values of water, absorption in \% in 2 and 24 hours, according to the species and the provenance of the material.

\begin{tabular}{|c|c|c|c|c|c|c|}
\hline \multirow{3}{*}{ Procedências } & \multicolumn{6}{|c|}{ Espécies } \\
\hline & \multicolumn{2}{|c|}{ E. cloeziana } & \multicolumn{2}{|c|}{ E. grandis } & \multicolumn{2}{|c|}{ E. saligna } \\
\hline & $2 \mathrm{~h}$ & $24 \mathrm{~h}$ & $2 \mathrm{~h}$ & $24 \mathrm{~h}$ & $2 \mathrm{~h}$ & $24 \mathrm{~h}$ \\
\hline 9785 & $61,05 \mathrm{~A}$ & $74,19 \mathrm{~A}$ & --- & --- & --- & --- \\
\hline 97852 & $61,62 \mathrm{~A}$ & $80,11 \mathrm{~A}$ & --- & --- & --- & --- \\
\hline 43 & --- & --- & $62,91 \mathrm{AB}$ & 88,99 ABC & --- & --- \\
\hline 45 & --- & --- & $45,67 \mathrm{ABC}$ & $74,08 \mathrm{BC}$ & --- & --- \\
\hline 47 & --- & --- & $58,72 \mathrm{AB}$ & $84,94 \mathrm{ABC}$ & --- & --- \\
\hline 48 & --- & --- & $55,20 \mathrm{AB}$ & $73,94 \mathrm{BC}$ & --- & --- \\
\hline 8535 & --- & --- & $64,99 \mathrm{~A}$ & $92,15 \mathrm{AB}$ & --- & --- \\
\hline 9753 & --- & --- & $52,75 \mathrm{ABC}$ & $77,62 \mathrm{ABC}$ & --- & --- \\
\hline 9789 & --- & --- & $39,73 \mathrm{BC}$ & $72,83 \mathrm{C}$ & --- & --- \\
\hline 10634 & --- & --- & $51,18 \mathrm{ABC}$ & $93,41 \mathrm{~A}$ & --- & --- \\
\hline 10695 & --- & --- & $34,88 \mathrm{C}$ & 77,76 ABC & --- & --- \\
\hline 10696 & --- & --- & $71,43 \mathrm{~A}$ & $86,96 \mathrm{ABC}$ & --- & --- \\
\hline 7785 & --- & --- & --- & --- & $45,04 \mathrm{~A}$ & $65,07 \mathrm{~A}$ \\
\hline 7808 & --- & --- & --- & --- & $50,09 \mathrm{~A}$ & $71,41 \mathrm{~A}$ \\
\hline 10698 & --- & --- & --- & --- & $41,86 \mathrm{~A}$ & $77,01 \mathrm{~A}$ \\
\hline Média Espécies ${ }^{1}$ & $61,34 \mathrm{a}$ & $77,15 \mathrm{ab}$ & $53,75 \mathrm{ab}$ & $82,27 \mathrm{a}$ & $45,66 \mathrm{~b}$ & $71,16 b$ \\
\hline $\mathrm{CV}(\%) 2 \mathrm{~h}$ & & & & & & \\
\hline $\mathrm{CV}(\%) 24 \mathrm{~h}$ & & & & & & \\
\hline
\end{tabular}

${ }^{1}$ Médias seguidas de mesma letra minúscula, na linha, e maiúscula, na coluna, não diferem entre si, pelo teste Tukey, a 5\% de significância.

Em pesquisa realizada por Iwakiri et al. (2001), para painéis de madeira aglomerada com cinco espécies de pinus, os valores médios obtidos foram na faixa de $68,82 \%$ a $83,60 \%$, para absorção de água, após 2 horas e de $78,69 \%$ a 93,81\%, para absorção de água após 24 horas. Os resultados obtidos para painéis produzidos com espécies de Pinus elliottii e Eucalyptus dunnii mostraram valores médios de absorção de água que variaram na faixa de $42,09 \%$ a $71,75 \%$, para duas horas de imersão e de $73,12 \%$ a $80,05 \%$, para 24 horas (IWAKIRI et al., 1996). Dessa forma, os valores obtidos por esta pesquisa ficaram muito próximos aos valores de literatura.

$\mathrm{Na}$ Tabela 4, estão apresentados os valores médios de inchamento em espessura em 2 e 24 horas, para espécies e procedências de eucalipto.

O inchamento em espessura, após 2 horas, não foi afetado pela espécie. Para as procedências de Eucalyptus cloeziana, observou-se o menor valor para a 97852, com 14,24\%. Para Eucalyptus grandis, destaque para as de número 43, 48, 10634 e 10695, com valores de 12,82, 13,27, 11,69 e $12,78 \%$, respectivamente. Esses valores foram iguais estatisticamente a de número 10696. A procedência 7785 de Eucalyptus saligna foi a que apresentou menor média em inchamento após 2 horas, com valor médio de 13,69\%, sendo estatisticamente igual a 10698 .

Para o inchamento em espessura após 24 horas, nota-se a mesma tendência entre as espécies estudadas, não apresentando diferenças significativas entre si. Entre as procedências de Eucalyptus cloeziana e Eucalyptus saligna, não foram observadas diferenças significativas entre suas médias. Para Eucalyptus grandis, manteve-se com menor valor médio de inchamento a de número 43 , com $18,76 \%$, sendo estatisticamente igual a $45,47,48$, 9753,9789, 10634 e 10695.

Cerne, Lavras, v. 17, n. 4, p. 443-452, out./dez. 2011 
Tabela 4 - Valores médios de inchamento em espessura, em \% em 2 e 24 horas, segundo as espécies e a procedência.

Table 4-Medium values in thickness swelling, in \% in 2 and 24 hours, according to species and provenances.

\begin{tabular}{|c|c|c|c|c|c|c|}
\hline \multirow{3}{*}{ Procedências } & \multicolumn{6}{|c|}{ Espécies } \\
\hline & \multicolumn{2}{|c|}{ E. cloeziana } & \multicolumn{2}{|c|}{ E. grandis } & \multicolumn{2}{|c|}{ E. saligna } \\
\hline & $2 \mathrm{~h}$ & $24 \mathrm{~h}$ & $2 \mathrm{~h}$ & $24 \mathrm{~h}$ & $2 \mathrm{~h}$ & $24 \mathrm{~h}$ \\
\hline 9785 & $17,32 \mathrm{~A}$ & $27,20 \mathrm{~A}$ & --- & --- & --- & --- \\
\hline 97852 & $14,24 \mathrm{~B}$ & $21,73 \mathrm{~A}$ & --- & --- & --- & --- \\
\hline 43 & --- & --- & $12,82 \mathrm{D}$ & $18,76 \mathrm{C}$ & --- & --- \\
\hline 45 & --- & --- & $17,98 \mathrm{ABC}$ & $23,93 \mathrm{BC}$ & --- & --- \\
\hline 47 & --- & --- & $21,51 \mathrm{~A}$ & $27,90 \mathrm{ABC}$ & --- & --- \\
\hline 48 & --- & --- & $13,27 \mathrm{D}$ & $21,62 \mathrm{BC}$ & --- & --- \\
\hline 8535 & --- & --- & $21,06 \mathrm{AB}$ & $38,17 \mathrm{~A}$ & --- & --- \\
\hline 9753 & --- & --- & $18,37 \mathrm{ABC}$ & $29,09 \mathrm{ABC}$ & --- & --- \\
\hline 9789 & --- & --- & $18,37 \mathrm{ABC}$ & $26,47 \mathrm{BC}$ & --- & --- \\
\hline 10634 & --- & --- & $11,69 \mathrm{D}$ & $21,88 \mathrm{BC}$ & --- & --- \\
\hline 10695 & --- & --- & $12,78 \mathrm{D}$ & $24,02 \mathrm{BC}$ & --- & --- \\
\hline 10696 & --- & --- & $17,15 \mathrm{BCD}$ & $31,59 \mathrm{AB}$ & --- & --- \\
\hline 7785 & --- & --- & --- & --- & $13,69 \mathrm{~B}$ & $22,78 \mathrm{~A}$ \\
\hline 7808 & --- & --- & --- & --- & $18,67 \mathrm{~A}$ & $29,32 \mathrm{~A}$ \\
\hline 10698 & --- & --- & --- & --- & $17,86 \mathrm{AB}$ & $29,36 \mathrm{~A}$ \\
\hline Média Espécies ${ }^{1}$ & $15,78 \mathrm{a}$ & $24,46 \mathrm{a}$ & $16,50 \mathrm{a}$ & $26,34 \mathrm{a}$ & $16,74 \mathrm{a}$ & $27,15 \mathrm{a}$ \\
\hline CV (\%) $2 \mathrm{~h}$ & \multicolumn{6}{|c|}{12,79} \\
\hline CV (\%) $24 \mathrm{~h}$ & \multicolumn{6}{|c|}{19,98} \\
\hline
\end{tabular}

${ }^{1}$ Médias seguidas de mesma letra minúscula, na linha, e maiúscula, na coluna, não diferem entre si, pelo teste Tukey, a 5\% de significância.

Os resultados de inchamento em espessura, após 2 e 24 horas de imersão, foram menores que aqueles encontrados por Iwakiri et al. (2001), estudando cinco espécies de pinus. Iwakiri et al. (2000), trabalhando com espécies de eucalipto oriundas do aproveitamento de serrarias, obtiveram, para essa propriedade, valores de $12,38 \%$ a $30,60 \%$, após 2 horas e de $23,50 \%$ a $38,81 \%$, após 24 horas.

A norma de comercialização CS 236-66 (CS, 1968) exige valores para essa propriedade física de, no máximo, $35 \%$ para painéis de média densidade e $30 \%$ para os de baixa densidade. Dessa forma apenas as procedências 8535 e 10696 de Eucalyptus grandis ultrapassaram este valor.

Portanto, as procedências e as espécies avaliadas neste trabalho apresentaram boas características de estabilidade dimensional, em virtude do material genético utilizado, da aplicação da emulsão de parafina e, principalmente, da densidade baixa do painel, que levou à consequente redução na razão de compactação, culminando com a melhora nas propriedades físicas do mesmo.

Os dados apresentados na Tabela 5 são referentes às tensões obtidas no teste de tração perpendicular (TP) e compressão paralela $(\mathrm{CP})$.

Observa-se que não houve diferenças significativas entre os valores médios de tração perpendicular, tanto entre espécies quanto entre procedências. Tal fato indica que a colagem do painel foi homogênea para todos os materiais genéticos testados.

Em trabalho realizado por Pedrazzi et al. (2006), com utilização de $8 \%$ de adesivo uréia-formaldeído e densidade de $0,65 \mathrm{~g} / \mathrm{cm}^{3}$ do painel, foram relatados valores de $1,75 \mathrm{kgf} / \mathrm{cm}^{2}$ e $1,35 \mathrm{kgf} / \mathrm{cm}^{2}$, com a utilização de resíduos de Eucalyptus saligna, gerados pela indústria de celulose, ficando abaixo dos obtidos por este trabalho. A norma de comercialização americana CS 236-66 (CS, 1968) para painéis de média densidade, com colagem à base de uréiaformaldeído, estabelece valores de $4,2 \mathrm{kgf} / \mathrm{cm}^{2}$ e para os de

Cerne, Lavras, v. 17, n. 4, p. 443-452, out./dez. 2011 
Tabela 5 - Valores médios de tração perpendicular $\left(\mathrm{kgf} / \mathrm{cm}^{2}\right)$ e resistência a compressão paralela $\left(\mathrm{kgf} / \mathrm{cm}^{2}\right)$.

Table 5-Medium values of perpendicular traction $\left(\mathrm{kgf} / \mathrm{cm}^{2}\right)$ and parallel compression $\left(\mathrm{kgf} / \mathrm{cm}^{2}\right)$.

\begin{tabular}{|c|c|c|c|c|c|c|}
\hline \multirow{3}{*}{ Procedências } & \multicolumn{6}{|c|}{ Espécies } \\
\hline & \multicolumn{2}{|c|}{ E. cloeziana } & \multicolumn{2}{|c|}{ E. grandis } & \multicolumn{2}{|c|}{ E. saligna } \\
\hline & TP & $\mathrm{CP}$ & $\mathrm{TP}$ & $\mathrm{CP}$ & $\mathrm{TP}$ & $\mathrm{CP}$ \\
\hline 9785 & $3,18 \mathrm{~A}$ & $58,02 \mathrm{~A}$ & --- & --- & -- & --- \\
\hline 97852 & $3,01 \mathrm{~A}$ & $66,64 \mathrm{~A}$ & --- & --- & --- & --- \\
\hline 43 & --- & --- & $2,53 \mathrm{~A}$ & $48,58 \mathrm{~A}$ & --- & --- \\
\hline 45 & --- & --- & $3,08 \mathrm{~A}$ & $59,73 \mathrm{~A}$ & --- & --- \\
\hline 47 & --- & --- & $2,80 \mathrm{~A}$ & $44,70 \mathrm{~A}$ & --- & --- \\
\hline 48 & --- & --- & $3,04 \mathrm{~A}$ & $58,49 \mathrm{~A}$ & --- & --- \\
\hline 8535 & --- & -- & $2,76 \mathrm{~A}$ & $46,69 \mathrm{~A}$ & --- & --- \\
\hline 9753 & --- & --- & $2,89 \mathrm{~A}$ & 49,37 A & --- & --- \\
\hline 9789 & --- & --- & $2,99 \mathrm{~A}$ & $53,26 \mathrm{~A}$ & --- & --- \\
\hline 10634 & --- & --- & $2,69 \mathrm{~A}$ & $53,20 \mathrm{~A}$ & --- & --- \\
\hline 10695 & --- & --- & $3,57 \mathrm{~A}$ & $55,95 \mathrm{~A}$ & --- & --- \\
\hline 10696 & --- & --- & $2,69 \mathrm{~A}$ & $58,41 \mathrm{~A}$ & --- & --- \\
\hline 7785 & --- & --- & --- & --- & $2,97 \mathrm{~A}$ & $53,69 \mathrm{~A}$ \\
\hline 7808 & --- & --- & --- & --- & $2,61 \mathrm{~A}$ & $58,97 \mathrm{~A}$ \\
\hline 10698 & --- & --- & --- & --- & $2,89 \mathrm{~A}$ & $52,34 \mathrm{~A}$ \\
\hline Média Espécies ${ }^{1}$ & $3,09 \mathrm{a}$ & $62,33 \mathrm{a}$ & $2,90 \mathrm{a}$ & $52,84 \mathrm{~b}$ & $2,82 \mathrm{a}$ & $55,00 \mathrm{ab}$ \\
\hline CV (\%) TP & \multicolumn{6}{|c|}{17,57} \\
\hline $\mathrm{CV}(\%) \mathrm{CP}$ & \multicolumn{6}{|c|}{20,32} \\
\hline
\end{tabular}

${ }^{1}$ Médias seguidas de mesma letra minúscula, na linha, e maiúscula, na coluna, não diferem entre si, pelo teste Tukey, a 5\% de significância

baixa densidade valor de no mínimo $2,10 \mathrm{kgf} / \mathrm{cm}^{2}$. Nesse sentido, apenas as procedências de número 43 e 10634 de Eucalyptus grandis atenderam às exigências da norma.

Para o teste de compressão, observou-se que não houve diferenças significativas entre as procedências nem entre as espécies. Nessas ultimas, observa-se maior valor médio de tensão na compressão para Eucalyptus cloeziana, sendo estatisticamente igual ao valor observado para Eucalyptus saligna.

Mendes (2008), trabalhando com misturas entre Eucalyptus urophylla e bagaço de cana-de-acúcar na confecção de painéis aglomerados, obteve resultados de compressão variando entre 45,60 e $51,07 \mathrm{kgf} / \mathrm{cm}^{2}$. Nesse sentido, os valores obtidos nesse experimento se mostraram acima da citada literatura.

Na Tabela 6 observam-se os valores de módulo de elasticidade (MOE) e módulo de ruptura (MOR) para os painéis aglomerados, produzidos a partir de diferentes procedências e espécies de eucalipto.
Pela análise dos resultados de MOE, verifica-se que não houve diferenças significativas entre as médias analisadas para as procedências de Eucalyptus cloeziana e Eucalyptus saligna, enquanto para Eucalyptus grandis foi observado maior valor médio para a de número 10695 , com $10537,8 \mathrm{kgf} / \mathrm{cm}^{2}$, sendo esta estatisticamente igual a 43, 45, 47, 48, 8535, 9789, 10634 e 10696.

Para as espécies, maior valor foi observado para Eucalyptus grandis, sendo estatisticamente igual ao valor encontrado para Eucalyptus cloeziana.

Iwakiri et al. (2000), utilizando resíduos de serraria na manufatura de aglomerados de eucalipto com $8 \%$ de adesivo uréia-formaldeído, obtiveram, para essa propriedade, valores de 17737,63 e $22068,85 \mathrm{kgf} /$ $\mathrm{cm}^{2}$. Pedrazzi et al. (2006) encontraram valores de MOE entre 10143 e $14774 \mathrm{kgf} / \mathrm{cm}^{2}$ para tratamento com $8 \%$ de adesivo, utilizando serragem de madeira de eucalipto. Os valores obtidos por esses estudos foram superiores aos deste trabalho. Da mesma forma, os resultados dessa

Cerne, Lavras, v. 17, n. 4, p. 443-452, out./dez. 2011 
Tabela 6 - Valores médios de MOE $\left(\mathrm{kgf} / \mathrm{cm}^{2}\right)$ e MOR $\left(\mathrm{kgf} / \mathrm{cm}^{2}\right)$, segundo as espécies e a procedência.

Table 6-Medium values of it MOE $\left(\mathrm{kgf} / \mathrm{cm}^{2}\right)$ and MOR $\left(\mathrm{kgf} / \mathrm{cm}^{2}\right)$, according to species and provenances.

\begin{tabular}{|c|c|c|c|c|c|c|}
\hline \multirow{3}{*}{ Procedências } & \multicolumn{6}{|c|}{ Espécies } \\
\hline & \multicolumn{2}{|c|}{ E. cloeziana } & \multicolumn{2}{|c|}{ E. grandis } & \multicolumn{2}{|c|}{ E. saligna } \\
\hline & MOE & MOR & MOE & MOR & MOE & MOR \\
\hline 9785 & $8.313,5 \mathrm{~A}$ & $166,5 \mathrm{~A}$ & --- & --- & --- & --- \\
\hline 97852 & $7.812,7 \mathrm{~A}$ & $139,2 \mathrm{~A}$ & --- & --- & --- & --- \\
\hline 43 & --- & --- & $7.710,1 \mathrm{AB}$ & $146,7 \mathrm{AB}$ & --- & --- \\
\hline 45 & --- & --- & $8.911,1 \mathrm{AB}$ & $196,8 \mathrm{AB}$ & --- & --- \\
\hline 47 & --- & --- & $8.682,4 \mathrm{AB}$ & $167,4 \mathrm{AB}$ & --- & --- \\
\hline 48 & --- & --- & $9.056,0 \mathrm{AB}$ & $193,8 \mathrm{AB}$ & --- & --- \\
\hline 8535 & --- & --- & $8.602,8 \mathrm{AB}$ & $175,5 \mathrm{AB}$ & --- & --- \\
\hline 9753 & --- & --- & $6.744,5 \mathrm{~B}$ & $135,0 \mathrm{~B}$ & --- & --- \\
\hline 9789 & --- & --- & $9.476,3 \mathrm{AB}$ & $184,6 \mathrm{AB}$ & --- & --- \\
\hline 10634 & --- & --- & $8.791,3 \mathrm{AB}$ & $177,8 \mathrm{AB}$ & --- & --- \\
\hline 10695 & --- & --- & $10.537,8 \mathrm{~A}$ & $230,1 \mathrm{~A}$ & --- & --- \\
\hline 10696 & --- & --- & $9.367,7 \mathrm{AB}$ & $183,0 \mathrm{AB}$ & --- & --- \\
\hline 7785 & --- & --- & --- & --- & $7.590,1 \mathrm{~A}$ & $150,7 \mathrm{~A}$ \\
\hline 7808 & --- & --- & --- & --- & $6.670,4 \mathrm{~A}$ & $127,2 \mathrm{~A}$ \\
\hline 10698 & --- & --- & --- & --- & $7.774,5 \mathrm{~A}$ & $164,4 \mathrm{~A}$ \\
\hline Média Espécies ${ }^{1}$ & $8.063,1 \mathrm{ab}$ & $152,8 \mathrm{~b}$ & $8.788,0 \mathrm{a}$ & $179,1 \mathrm{a}$ & $7.345,0 \mathrm{~b}$ & $147,5 \mathrm{~b}$ \\
\hline CV (\%) MOE & & & & 9,16 & & \\
\hline CV $(\%) \mathrm{MOR}$ & & & & 9,92 & & \\
\hline
\end{tabular}

${ }^{1}$ Médias seguidas de mesma letra minúscula, na linha, e maiúscula, na coluna, não diferem entre si, pelo teste Tukey, a 5\% de significância.

propriedade se mostraram abaixo das exigências da norma de comercialização americana CS 236-66 (CS, 1968) que exige valores mínimos de $10.500 \mathrm{kgf} / \mathrm{cm}^{2}$ para painéis de baixa densidade e $24500 \mathrm{kgf} / \mathrm{cm}^{2}$ para os de média densidade.

Para MOR não houve diferenças significativas entre as médias, dentro das procedências de Eucalyptus cloeziana e Eucalyptus saligna; já para Eucalyptus grandis, maior valor foi encontrado para 10695 , com $230,1 \mathrm{kgf} / \mathrm{cm}^{2}$, sendo estatisticamente diferente a procedência 9753. Quanto às espécies, pode-se inferir que a mais resistente foi a de Eucalyptus grandis, com $179,1 \mathrm{kgf} / \mathrm{cm}^{2}$.

Iwakiri et al. (2001), produzindo aglomerados a partir de madeira de diferentes espécies de pinus, encontraram valores de MOR à flexão entre 171,83 e $215,42 \mathrm{kgf} / \mathrm{cm}^{2}$. Iwakiri et al. (2000), trabalhando com resíduos de serraria gerados por madeira de diferentes espécies de eucalipto, encontraram valores de MOR entre
74,13 e $158,54 \mathrm{kgf} / \mathrm{cm}^{2}$. A norma de comercialização CS 236-66 (CS, 1968) coloca valores mínimos para essa propriedade de $98 \mathrm{kgf} / \mathrm{cm}^{2}$, para painéis aglomerados confeccionados com uréia-formaldeído e de baixa densidade. Para os de média densidade, a exigência passa a ser de $112 \mathrm{kgf} / \mathrm{cm}^{2}$. Os resultados observados no presente trabalho ficaram próximos aos encontrados na literatura e acima daqueles referenciados na norma.

\section{CONCLUSÕES}

Com base nos resultados obtidos nesta pesquisa, as seguintes conclusões podem ser apresentadas:

- de maneira geral os resíduos gerados pela laminação da madeira de eucalipto se apresentam com potencial para produção de painéis aglomerados;

- a espécie de Eucalyptus saligna apresentou melhor desempenho na absorção de água;

Cerne, Lavras, v. 17, n. 4, p. 443-452, out./dez. 2011 
- Eucalyptus cloeziana e Eucalyptus saligna se destacaram no teste de compressão;

- a espécie de Eucalyptus grandis apresentou melhor desempenho no que se refere a MOE e MOR, sendo que sua procedência de número 10695 se destacou para essas propriedades;

- de forma geral, com base nas propriedades físicas e mecânicas dos painéis, a procedência 10.695 e as espécies de Eucalyptus grandis foram as que apresentaram maiores potenciais para a produção de aglomerados.

\section{AGRADECIMENTOS}

À FAPEMIG - Fundação de Amparo a Pesquisa de Minas Gerais.

\section{REFERÊNCIAS}

AMERICAN SOCIETY FOR TESTING AND MATERIALS. Annual book of ASTM: D-1037-34.22: standard methods of evaluating properties of wood-base fiber and particles materials. Philadelphia, 2002.

AMERICAN SOCIETY FOR TESTING AND MATERIAL. Standard methods of evaluating the properties of wood-base fiber and particle panel materials. In: . Annual book of ASTM standard: ASTM D 1037-28. Philadelphia, 1982.

BRITO, E.O.; SAMPAIO, L. C.; OLIVEIRA, J. N.; BATISTA, D. C. Chapas de madeira aglomerada utilizando partículas oriundas de madeira maciça e de maravalhas. Scientia Forestalis, Piracicaba, n. 72, p. 17-21, dez. 2006.

BUFALINO, L. Avaliação da permeabilidade e sua influencia sobre as propriedades físicas e de colagem em painéis aglomerados. 2010. 76 p. Dissertação (Mestrado) Universidade Federal de Lavras, Lavras, 2010.

CABRAL, C. P.; VITAL, B. R.; DELLA LUCIA, R. M.; PIMENTA, A. S. Propriedade de chapas de aglomerado confeccionados com mistura de partículas de Eucalyptus spp e Pinus elliotti. Revista Árvore, Viçosa, v. 31, n. 5, p. 100-107, set./out. 2007.

CLOUTIER, A. Oriented stranboard (osb): raw material, manufacturing process, properties of wood-base fiber and particle materials. In: INTERNATIONAL SEMINAR ON SOLID WOOD PRODUCTS OF HIGH TECHNOLOGY, 1., 1998, Belo Horizonte. Anais... Belo Horizonte: SIF, 1998. p. 173-185.
COMMERCIAL STANDARD. Mat formed wood particle board: CS -236-66. Wallingford, 1968.

\section{DEUTSCHES INSTITUT FUR NORMUNG. Testing of} wood chipboards, bending test, determination of bending strength: DIN 52362. Berlin, 1982. 40 p.

FAGUNDES, H. A. V. Diagnóstico da produção de madeira serrada e geração de resíduos do processamento de madeira de florestas plantadas no Rio Grande do Sul. 2003. 180 p. Dissertação (Mestrado em Engenharia Civil) - Universidade Federal do Rio Grande do Sul, Porto Alegre, 2003.

FOOD AND ACRICULTURE ORGANIZATION OF UNITED NATIONS. Disponível em: < http://www.fao.org $>$. Acesso em: 1 fev. 2011.

IWAKIRI, S. et al. Produção de chapas de madeira aglomerada de cinco espécies de pinus tropicais. Floresta e Ambiente, Seropédica, v. 8, n. 1, p. 137-142, jan./dez. 2001.

IWAKIRI, S. et al. Produção de chapas de partículas de madeira de Pinus elliottii e Eucalyptus dunnii. Agrárias, Curitiba, v. 15, p. 34-41, 1996.

IWAKIRI, S. et al. Resíduos de serrarias na produção de painéis de madeira aglomerada de eucalipto. Scientia Agraria, Curitiba, v. 1, n. 1/2, p. 23-28, 2000.

KELLY, M. W. A critical literature review of relationships between processing parameters and physical properties of particleboards. Madison: U.S. Forest Production Laboratory, 1977. 66 p. (General Technical Report FPL, 10).

KUBLER, H. Wood as building and hobby material: how to use lumber, wood-base panels, and roundwood wisely in construction, for furniture, and as fuel. New York: Wiley, 1980. 256 p.

MALONEY, T. M. Modern particleboard e dry-process fiberboard manufacturing. 2. ed. San Francisco: M. Freeman, 1993. 689 p.

MENDES, R. F. Utilização do bagaço de cana de alambique na produção de painéis aglomerados. 2008 . 104 p. Monografia (Graduação em Engenharia Florestal) Universidade Federal de Lavras, Lavras, 2008.

Cerne, Lavras, v. 17, n. 4, p. 443-452, out./dez. 2011 
MOSLEMI, A. A. Particleboard. Carbondale: Southern Illinois University, 1974.

PEDRAZZI, C. et al. Qualidade de chapas de partículas de madeira aglomerada fabricadas com resíduos de uma indústria de celulose. Ciências Florestais, Santa Maria, v. 16, n. 2, p. 201-212, 2006.

TSOUMIS, G. Science and technology of wood: structure, properties, utilization. New York: Chapman \& Hall, 1991. 494 p.

Cerne, Lavras, v. 17, n. 4, p. 443-452, out./dez. 2011 